\title{
GRAPH-REGULARIZED COUPLED SPECTRAL UNMIXING FOR MULTISENSOR TIME-SERIES ANALYSIS
}

\author{
Naoto Yokoya ${ }^{1,2,3}$, Xiao Xiang Zhu ${ }^{1,2}$, and Antonio Plaza ${ }^{4}$ \\ ${ }^{1}$ Remote Sensing Technology Institute (IMF), German Aerospace Center (DLR), Germany \\ ${ }^{2}$ Signal Processing in Earth Observation (SiPEO), Technische Universität München (TUM), Germany \\ ${ }^{3}$ Department of Advanced Interdisciplinary Studies, University of Tokyo, Japan \\ ${ }^{4}$ Department of Technology of Computers and Communications, University of Extremadura, Spain
}

\begin{abstract}
A new methodology that solves unmixing problems involving a set of multisensor time-series spectral images is proposed in order to understand dynamic changes of the surface at a subpixel scale. The proposed methodology couples multiple unmixing problems via regularization on graphs between the multisensor time-series data to obtain robust and stable unmixing solutions beyond data modalities owing to different sensor characteristics and the effects of non-optimal atmospheric correction. A synthetic dataset that includes seasonal and trend changes on the surface and the residuals of nonoptimal atmospheric correction is used for numerical validation. Experimental results demonstrate the effectiveness of the proposed methodology.
\end{abstract}

Index Terms - Coupled spectral unmixing, multisensor data fusion, time-series analysis, change detection

\section{INTRODUCTION}

A synergetic use of hyperspectral and multispectral images is important for upcoming spaceborne imaging spectroscopy missions [1]. Spaceborne imaging spectroscopy enables monitoring dynamic processes of the surface in detail on a global scale; however, revisit time is limited compared to multispectral imaging satellites. Understanding of the dynamics on the surface can be improved by synergistically analyzing a time series of spaceborne hyperspectral and multispectral images.

In recent years, particular attention has been paid to spectral unmixing of multitemporal hyperspectral data owing to its ability to detect class-specific changes at a subpixel level [2][11]. Previous research has shown the effectiveness of multitemporal spectral unmixing in a wide range of applications [2]-[6]. Current research is focused on applying spectrallibrary-based unmixing methods to multitemporal unmixing

This research has been partly supported by Alexander von Humboldt Fellowship for postdoctoral researchers, Helmholtz Young Investigators Group "SiPEO” (VH-NG-1018, www.sipeo.bgu.tum.de), and Japan Society for the Promotion of Science (JSPS) KAKENHI 15K20955. problems [5, 9]. A data-driven approach has also been developed in $[10,11]$.

To the authors' knowledge, very few publications can be found in the literature that investigate time-series spectral unmixing from the viewpoint of synergistically exploiting hyperspectral and multispectral images. Hyperspectral and multispectral image fusion has received great attention to generate high-resolution hyperspectral data [12]; however, studies on time-series analysis are still lacking. To tackle spectral unmixing of a multisensor time series, it is challenging to determine how to use hyperspectral and multispectral images in complementary fashion. Furthermore, most of the previous studies on multitemporal hyperspectral unmixing do not take into account possible data mismatches due to non-optimal atmospheric correction, even though it always appears in timeseries spectral data [13]-[15].

In this paper, a novel methodology for spectral unmixing of multisensor time-series data is proposed. The objective is to understand dynamic changes on the surface at a subpixel scale from multisensor time-series data, beyond data modalities, due to different sensor characteristics and the effects of non-optimal atmospheric correction. Each spectral unmixing problem is solved cooperatively with those related to other images that have similar surface conditions to obtain a robust, stable, and accurate solution. Multiple unmixing problems are coupled via regularization on graphs between neighborhood images.

The remainder of the paper is organized as follows: Section II introduces our methodology. Experimental results on synthetic data are presented in Sections III. Section IV concludes the paper.

\section{METHODOLOGY}

To obtain robust and stable unmixing beyond data modalities due to different spectral characteristics and non-optimal atmospheric correction, we introduce coupled spectral unmixing for time-series analysis of multisensor spectral data, which solves an unmixing problem of a single image cooperatively 


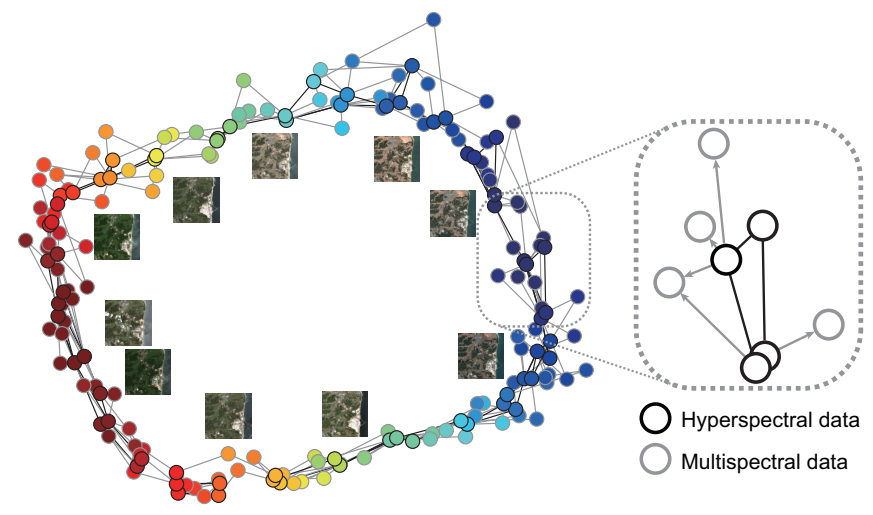

Fig. 1. Manifold of multisensor time-series spectral data

with those of its neighboring images (in the time-series hierarchy) by adopting an intrinsic manifold of the time-series dataset. Fig. 1 illustrates the manifold of multisensor timeseries spectral data. Each point represents a single image. Owing to changes on the surface, the multisensor time-series dataset forms the manifold, where neighborhood images have similar conditions of the surface. Coupled spectral unmixing of multisensor time-series data is formulated as a decentralized optimization problem for multi-agent systems [16], where each spectral unmixing is solved via a local optimization problem based only on information concerning other spectral unmixing problems in its neighborhood.

The multiple spectral unmixing problems are coupled via regularization on local graphs. In a local graph, a target image and its neighbors are regarded as nodes and connected by edges. Local graphs can be mixtures between undirected and directed graphs, depending on the difference in the spectral resolution between the two images. Undirected graphs are used between images with similar spectral resolution so that local spectral unmixing problems mutually influence each other. Hyperspectral imaging enables more accurate spectral unmixing than multispectral imaging. If the target image is a multispectral data set, hyperspectral neighboring images can improve the accuracy of the spectral unmixing for the target image; however, the reverse is not necessarily the case. Therefore, directed graphs, where the edges have a direction from higher-spectral-resolution data to lower-spectral-resolution data in the manifold, are also considered (Fig. 1).

Let us consider $K+1$ images $\left\{\mathbf{Y}_{k} \in \mathbb{R}^{B_{k} \times P}\right\}_{k=1}^{K+1}$, i.e., a target image and its $\mathrm{K}$-nearest neighbors. The numbers of spectral bands satisfy $B_{1} \leq B_{2} \leq \ldots \leq B_{K+1}$ without loss of generality. For each spectral unmixing, $L_{1 / 2}$ sparsity regularization on abundances is adopted []. It is supposed that the spectral signature matrix is obtained either from a spectral library or from the data itself. The optimization problem of coupled spectral unmixing can be formulated as

$$
\begin{aligned}
& \min _{\mathbf{X}, \mathbf{Z}} \frac{1}{2} \sum_{k=1}^{K+1}\left\|\mathbf{Y}_{k}-\mathbf{A}_{k} \mathbf{X}_{k}^{T}\right\|_{F}^{2} \\
& \quad+\frac{1}{2} \alpha \sum_{p=1}^{K} \sum_{q=p+1}^{K+1} \sum_{j, l=1}^{P}\left((\mathbf{G})_{p q}(\mathbf{G})_{q p}\left\|\mathbf{X}_{p}^{j}-\mathbf{X}_{q}^{l}\right\|_{2}^{2}\right. \\
& \left.\left.\quad+\left((\mathbf{G})_{q p}-(\mathbf{G})_{p q}\right)\left\|\mathbf{X}_{p}^{j}-\mathbf{Z}_{q}^{l}\right\|_{2}^{2}\right)\left(\mathbf{W}_{p q}\right)_{j l}\right) \\
& \text { s.t. } \quad \mathbf{X}_{k} \succeq 0, \mathbf{X}_{k}=\mathbf{Z}_{k},
\end{aligned}
$$

where $\mathbf{X}=\left[\mathbf{X}_{1}^{T}, \ldots, \mathbf{X}_{K+1}^{T}\right]^{T}, \mathbf{X}_{k} \in \mathbb{R}^{P \times M}$ denotes the abundance matrix of the $k$ th data $\left(\mathbf{Y}_{k}\right)$. The penalty term with the parameter $\alpha$ is the graph regularization, which places the restriction such that, if two spectral signatures are similar, the abundance vectors are also similar to each other. $\mathbf{W}_{p q} \in$ $\mathbb{R}^{P_{p} \times P_{q}}$ is the weight matrix on the graph between the $p$ th and $q$ th data, and $\left(\mathbf{W}_{p q}\right)_{j l}$ represents the closeness of two points: the $j$ th column of $\mathbf{Y}_{p}$ and the $l$ th column of $\mathbf{Y}_{q} . \mathbf{W}_{p q}$ is designated by the spectral similarity measurement using a distance metric, such as the Euclidean distance or spectral angle distance (SAD). $\mathbf{G} \in \mathbb{R}^{K+1 \times K+1}$ is the adjacency matrix that represents the local graph between $K+1$ images. $\mathbf{G}_{p q}=\mathbf{G}_{q p}=1$ if the $p$ th and $q$ th images are connected by the undirected edge, whereas $\mathbf{G}_{p q}=0$ and $\mathbf{G}_{q p}=1$ if they are connected by the directed edge from the $q$ th image to the $p$ th image. $\mathbf{Z}=\left[\mathbf{Z}_{1}^{T}, \ldots, \mathbf{Z}_{K+1}^{T}\right]^{T}$ is auxiliary variables that implement the directed-graph regularization.

The formula in (1) can be solved by alternating optimization after the initialization of $\mathbf{X}$ and $\mathbf{Z}$ using a conventional method for individual spectral unmixing, such as the constrained least squares (CLS) method [17]. The alternating update rules (2)-(4) are obtained by the augmented Lagrange method. $\boldsymbol{\Theta}_{k} \succ \mathbf{0}$ denotes the augmented Lagrangian parameters. $\mathbf{D}_{p q_{1}} \in \mathbb{R}^{P_{q} \times P_{q}}$ and $\mathbf{D}_{p q_{2}} \in \mathbb{R}^{P_{p} \times P_{p}}$ denote diagonal matrices whose entries are column and row sums of $\mathbf{W}_{p q}$, respectively. The simplest way to construct $\mathbf{W}_{p q}$ is to calculate the spectral similarity between only a pair of spatially corresponding pixels. In this case, $\mathbf{W}_{p q}=\mathbf{D}_{p q 1}=\mathbf{D}_{p q 2}$ if $P_{p}=P_{q}$ is satisfied. We use this technique to save computational costs. The average of non-zero elements in $\mathbf{W}$ is used to find the $K$-nearest neighbors and construct $\mathbf{G}$, which is referred to as manifold-based coupling. An alternative means of constructing $\mathbf{G}$ is the use of sequential coupling that uses temporally neighboring images. Note that the problem (1) does not include the abundance sum-to-one constraint. To satisfy the abundance sum-to-one constraint, a method given in [17] is adopted.

The proposed algorithm is summarized in Algorithm 1. 


$$
\begin{aligned}
\left(\mathbf{X}_{k}\right)_{j m} & \leftarrow\left(\mathbf{X}_{k}\right)_{j m} \frac{\left(\mathbf{Y}_{k}^{T} \mathbf{A}_{k}+\alpha\left(\sum_{p=1}^{k-1} \mathbf{G}_{p k} \mathbf{G}_{k p} \mathbf{W}_{p k}^{T} \mathbf{X}_{p}+\sum_{q=k+1}^{K+1}\left((\mathbf{G})_{k q}(\mathbf{G})_{q k} \mathbf{W}_{k q} \mathbf{X}_{q}+\left((\mathbf{G})_{q k}-(\mathbf{G})_{k q}\right) \mathbf{W}_{k q} \mathbf{Z}_{q}\right)\right)+\boldsymbol{\Theta}_{k}\right)_{j m}}{\left.\left(\mathbf{X}_{k} \mathbf{A}_{k}^{T} \mathbf{A}_{k}+\alpha\left(\sum_{p=1}^{k-1}(\mathbf{G})_{p k}(\mathbf{G})_{k p} \mathbf{D}_{p k 2} \mathbf{X}_{k}+\sum_{q=k+1}^{K+1}(\mathbf{G})_{k q}(\mathbf{G})_{q k}+(\mathbf{G})_{q k}-(\mathbf{G})_{k q}\right) \mathbf{D}_{k q 1} \mathbf{X}_{k}\right)\right)_{j m}} \\
\left(\mathbf{Z}_{k}\right)_{j m} & \leftarrow\left(\mathbf{Z}_{k}\right)_{j m} \frac{\alpha\left(\sum_{p=1}^{k-1}\left((\mathbf{G})_{k p}-(\mathbf{G})_{p k}\right) \mathbf{W}_{p k}^{T} \mathbf{X}_{p}+\rho \mathbf{X}_{k}\right)_{j m}}{\left(\alpha \sum_{p=1}^{k-1}\left((\mathbf{G})_{k p}-(\mathbf{G})_{p k}\right) \mathbf{D}_{p k 2} \mathbf{Z}_{k}+\boldsymbol{\Theta}_{k}+\rho \mathbf{Z}_{k}\right)_{j m}} \\
\left(\boldsymbol{\Theta}_{k}\right)_{j m} & \leftarrow\left(\boldsymbol{\Theta}_{k}\right)_{j m}+\rho\left(\mathbf{Z}_{k}-\mathbf{X}_{k}\right)_{j m}
\end{aligned}
$$

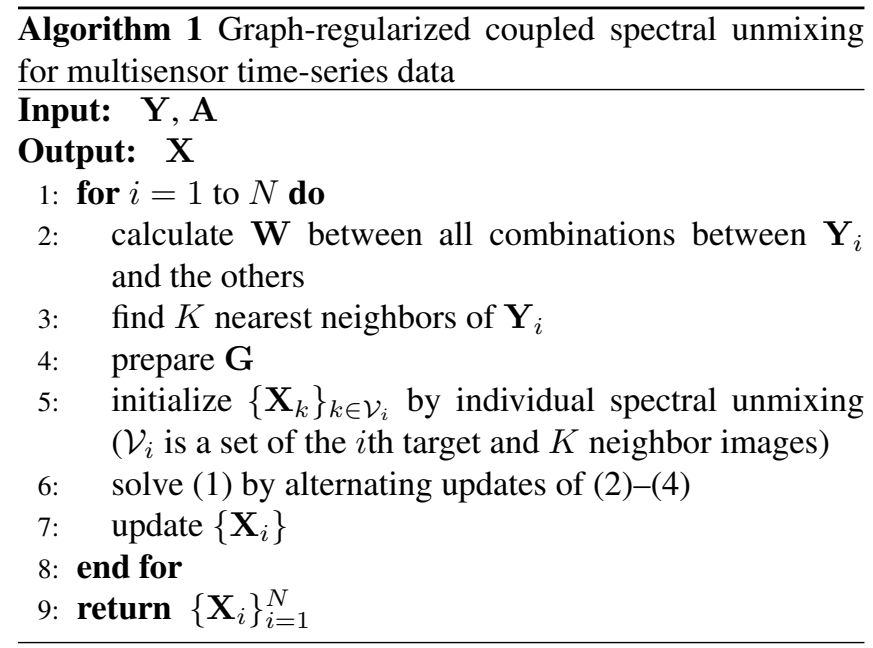

\section{EXPERIMENT}

\subsection{Synthetic multisensor time-series spectral images}

A set of synthetic multisensor time-series spectral images was used to numerically validate the proposed methodology. The synthetic dataset was simulated using the abundance maps of the Fractal 1 synthetic data [18]. The Fractal 1 image is composed of nine endmembers; their abundance maps were generated from fractal patterns with the size of $100 \times 100$ pixels. To simulate realistic changes on the surface, seasonal changes and trend changes were considered. Nine endmembers were selected from the U.S. Geological Survey (USGS) spectral library: grass, dry grass, oak, soil, melting snow, water, asphalt, green house, and concrete. Seasonal changes were simulated for grass, dry grass, oak, soil, and melting snow using periodic functions, and trend changes are simulated for green house, and concrete using a monotonic increasing function.

The spectral response functions of AVIRIS (bands 1-32, 36-96, 100-160, and 163-224) and Landsat-8 (bands 1-8) were used to simulate hyperspectral and multispectral images, respectively. Additive Gaussian noise with a signal-to-noise ratio (SNR) of 100 was added to each temporal image. Nonoptimal atmospheric correction was simulated using a Gaussian random vector for the residual gains. The revisit cycles of the hyperspectral and multispectral imagers were set to 16 and 27 days, respectively, which correspond to those of Landsat-8 and EnMAP. Two scenarios are considered for data acquisi- tion: 1) full acquisition, that assumes a clear sky for all images, and 2) realistic acquisition, that assumes a clear sky for $30 \%$ of the observations. The total observation period is set to five years and therefore the total number of images is 68 and 115 for hyperspectral and multispectral images, respectively. Composite color images of five examples are shown in Fig. 2(a). The corresponding reference abundances of grass and concrete are presented in the first row images of Fig. 2(b)(c).

\subsection{Experimental results}

We use three methods: 1) individual spectral unmixing (ISU) based on the CLS method; 2) coupled spectral unmixing with sequential coupling (CSU 1); and 3) coupled spectral unmixing with manifold-based coupling (CSU 2). To investigate the efficacy of using multiple sensors, these methods are performed on three different datasets: 1) only hyperspectral images; 2) only multispectral images; and 3) hyperspectral and multispectral images. A is given, $K=2$, and $\alpha=1$.

Table 1 shows the average root-mean-square errors (RMSEs) of abundances, defined by $\frac{1}{N} \sum_{i=1}^{N}\left\|\mathbf{X}_{i}-\tilde{\mathbf{X}}_{i}\right\|_{F}$. For the realistic data acquisition scenario, the average results of 10 Monte Carlo trials obtained after selecting 30\% of the observations are shown. Note that only the unmixing accuracy of multispectral images is shown for the third dataset, because only multispectral images can gain benefits, whereas the results of hyperspectral images are the same as for the first dataset. The coupled spectral unmixing algorithms outperform individual spectral unmixing for the first and third datasets. The unmixing accuracy of multispectral data is highly improved owing to the support of neighboring hyperspectral images. Coupled spectral unmixing based on manifold-based coupling outperforms that based on sequential coupling in many cases, particularly in the realistic data acquisition scenarios. This suggests that manifold-based coupling leads to more accurate and robust unmixing results than sequential coupling. Coupled spectral unmixing with sequential coupling performs well in ideal scenarios because sequential neighbors with high temporal resolution represent neighbors in the manifold of time-series data well. In contrast, if data acquisition is limited to realistic scenarios, manifold-based coupling is required because sequential neighbors do not always represent neighbors in the manifold.

Figs. 2(b)(c) present reference abundance maps of grass and concrete for the five examples of the third dataset with 
Table 1. Average RMSEs of abundances obtained before atmospheric normalization from three datasets of synthetic time-series spectral images, i.e. only hyperspectral (HS) data (68 images), only multispectral (MS) data (115 images), and both HS and MS data (183 images) using ISU, CSU 1, and CSU 2. RMSEs for only MS data are shown for the last dataset.

\begin{tabular}{c|cc|cc|cc}
\hline Dataset & \multicolumn{2}{|c|}{ HS } & \multicolumn{2}{c|}{ MS } & \multicolumn{2}{c}{ MS (coupled with HS) } \\
\hline Acquisition & Ideal & Realistic & Ideal & Realistic & Ideal & Realistic \\
\hline ISU & 0.028181 & 0.028526 & $\mathbf{0 . 1 1 4 8 4}$ & $\mathbf{0 . 1 1 7 1 9}$ & 0.11484 & 0.11719 \\
CSU 1 & $\mathbf{0 . 0 2 5 2 6 8}$ & 0.026195 & 0.1161 & 0.12110 & 0.086309 & 0.092507 \\
CSU 2 & 0.025552 & $\mathbf{0 . 0 2 5 8 9 6}$ & 0.11538 & 0.11840 & $\mathbf{0 . 0 4 9 2 0 7}$ & $\mathbf{0 . 0 5 2 0 1 6}$ \\
\hline
\end{tabular}

(a)

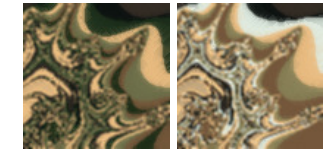

(b)

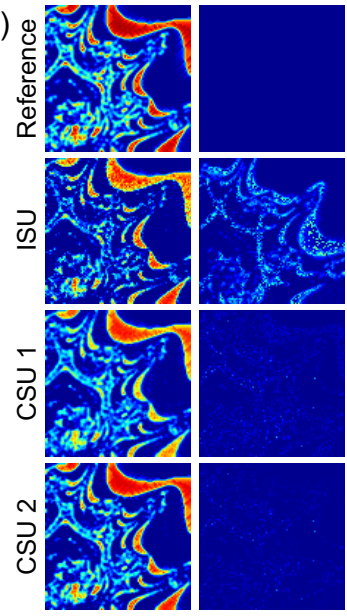

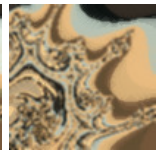
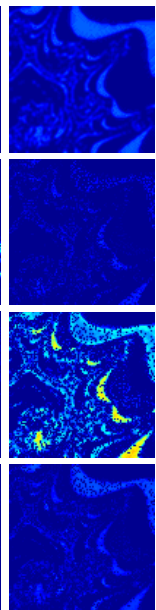
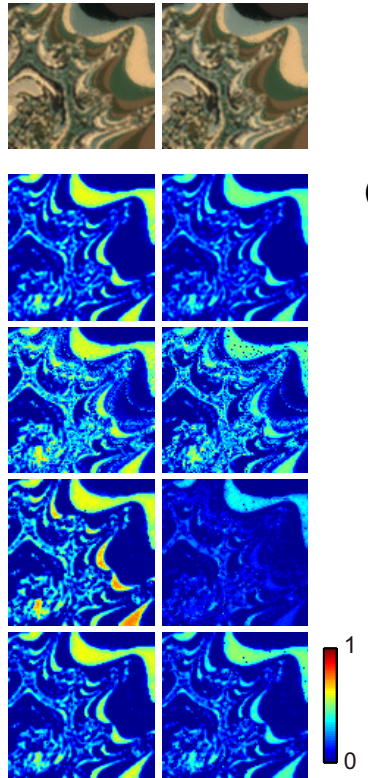

(c)

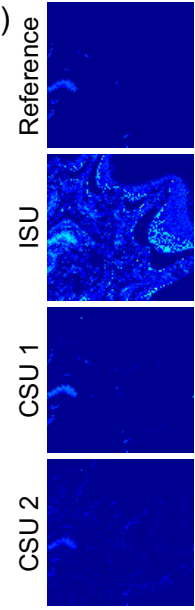

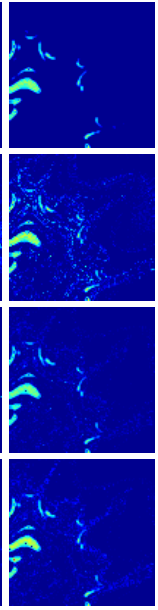
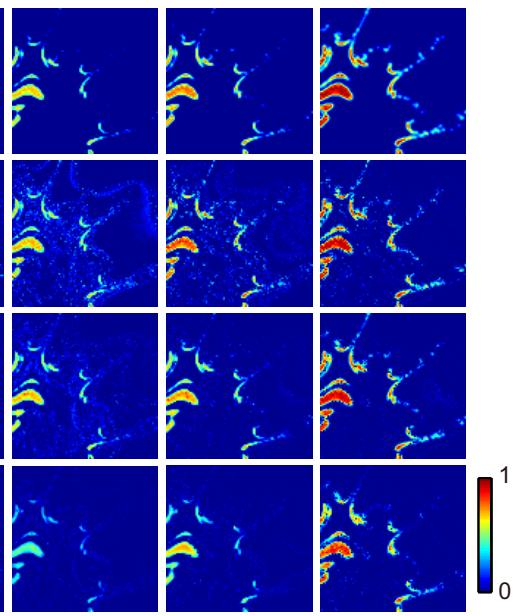

Fig. 2. (a) Color composites of ten images in the synthetic dataset and their corresponding abundance maps of (b) grass and (c) concrete. Abundances of reference and estimated abundances obtained by ISU, CSU 1, and CSU 2 are shown from top to bottom in (b) and (c).

one trial of the realistic acquisition scenario and the corresponding estimated abundance maps obtained by the three methods. Serial day numbers of the subset are 433, 1009, 1313,1473 , and 1825 , which are all simulated as observed by the multispectral imager. In Fig. 2(b), the coupled spectral unmixing methods shows better resemblance to the reference compared to individual spectral unmixing. In particular, CSU 2 exhibits the best results. On the other hand, in Fig. 2(c), CSU 1 shows the best resemblance, whereas both CSU 1 and CSU 2 outperform individual spectral unmixing. These results imply that manifold-based coupling better captures seasonal changes and sequential coupling is more suitable for detection of trend changes.

\section{CONCLUSIONS}

A coupled spectral unmixing methodology was proposed for multisensor time-series analysis. The proposed methodology provides robust and stable unmixing solutions beyond data modalities due to the different spectral characteristics of imagers and the effects of non-optimal atmospheric correction. A single unmixing problem is cooperatively solved with other unmixing problems in its neighborhood in the manifold of the time-series dataset. Multiple unmixing problems are coupled via regularization on graphs between the local images. Numerical validation was performed with synthetic time-series data composed of hyperspectral and multispectral images, including trend and seasonal changes on the surface and residual gains simulating non-optimal atmospheric correction. The experiment demonstrated the effectiveness of the coupled spectral unmixing algorithm compared to individual spectral unmixing. A generalization of the proposed methodology and its application to real data is noteworthy [19].

\section{REFERENCES}

[1] L. Guanter, H. Kaufmann, K. Segl, S. Foerster, C. Rogass, S. Chabrillat, T. Kuester, A. Hollstein, G. Ross- 
ner, C. Chlebek, C. Straif, S. Fischer, S. Schrader, T. Storch, U. Heiden, A. Müller, M. Bachmann, H. Mühle, R. Müller, M. Habermeyer, A. Ohndorf, J. Hill, H. Buddenbaum, P. Hostert, S. van der Linden, P. J. Leitão, A. Rabe, R. Doerffer, H. Krasemann, H. Xi, W. Mauser, T. Hank, M. Locherer, M. Rast, K. Staenz, and B. Sang, "The EnMAP spaceborne imaging spectroscopy mission for Earth observation," Remote Sensing, vol. 7, pp. 8830-8857, 2015.

[2] D. Lu, M. Batistella, and E. Moran, "Multitemporal spectral mixture analysis for Amazonian land-cover change detection," Can. J. Remote Sens., vol. 30, no. 1, pp. 87-100, 2004.

[3] D. B. Lobell and G. P. Asner, "Cropland distributions from temp oral unmixing of MODIS data," Remote Sens. Environ., vol. 93, no. 3, pp. 412-422, Nov. 2004.

[4] M. A. Goenaga, M. C. Torres-Madronero, M. VelezReyes, S. J. Van Bloem, and J. D. Chinea, "Unmixing analysis of a time series of Hyperion images over the Guánica dry forest in Puerto Rico," IEEE J. Sel. Topics Appl. Earth Observ. Remote Sens., vol. 6, no. 2, pp. 329-338, Apr. 2013.

[5] B. Somers and G. P. Asner, "Multi-temporal hyperspectral mixture analysis and feature selection for invasive species mapping in rainforests," Remote Sens. Environ., vol. 136, pp. 14-27, Sep. 2013.

[6] P. Du, S. Liu, P. Liu, K. Tan, and L. Cheng, "Subpixel change detection for urban land-cover analysis via multi-temporal remote sensing images," Geo-spatial Inf. Sci., vol. 17, no. 1, pp. 26-38, 2014.

[7] Q. Du, L. Wasson, and R. King, "Unsupervised linear unmixing for change detection in multitemporal airborne hyperspectral imagery," in Proc. IEEE Int. Workshop MultiTemp, 2005, pp. 136-140.

[8] A. Ertürk and A. Plaza, "Informative change detection by unmixing for hyperspectral images," IEEE Geosci. Remote Sens. Lett., vol. 12, no. 6, pp. 1252-1256, Jun. 2015.

[9] A. Ertürk, M.-D. Iordache, and A. Plaza, "Sparse unmixing based change detection for multi-temporal hyperspectral images," IEEE J. Sel. Topics Appl. Earth Observ. Remote Sens., in press.

[10] N. Yokoya and X. X. Zhu, "Graph regularized coupled spectral unmixing for change detection," Proc. WHISPERS, Tokyo, Japan, June 2-5, 2015.

[11] S. Liu, L. Bruzzone, F. Bovolo, and P. Du, "Unsupervised multitemporal spectral unmixing for detecting multiple changes in hyperspectral images," IEEE Trans. Geosci. Remote Sens., in press
[12] N. Yokoya, T. Yairi, and A. Iwasaki, "Coupled nonnegative matrix factorization unmixing for hyperspectral and multispectral data fusion," IEEE Trans. Geosci. Remote Sens., vol. 50, no. 2, pp. 528-537, 2012.

[13] A. Singh, "Digital change detection techniques using remotely-sensed data," Int. J. Remote Sens., vol. 10, no. 6, pp. 989-1003, 1989.

[14] C. Songh, C. E. Woodcock, K. C. Seto, M. P. Lenney, and S. A. Macomber, "Classification and change detection using Landsat TM data: when and how to correct atmospheric effects?" Remote Sens. Environ., vol. 75, pp. 230-244, 2001.

[15] P. Coppin, I. Jonckheere, K. Nackaerts, B. Muys, and E. Lambin, "Digital change detection methods in ecosystem monitoring: a review," Int. J. Remote Sens., vol. 25, no. 9 , pp. $1565-1596$.

[16] S. L. Waslander, G. Inalhan and C. J. Tomlin, Decentralized Optimization via Nash Bargaining, 2004, Kluwer Academic Press.

[17] D. C. Heinz and C.-I. Chang, "Fully constrained least squares linear spectral mixture analysis method for material quantification in hyperspectral imagery," IEEE Trans. Geosci. Remote Sens., vol. 39, no. 3, pp. 529545, 2001.

[18] J. Plaza, E. M. T. Hendrix, I García, G. Martín, A. Plaza, "On endmember identification in hyperspectral images without pure pixels: A comparison of algorithms," $J$. Math. Imaging Vis., vol. 42, pp. 163-175, 2012.

[19] N. Yokoya, X. X. Zhu, and A. Plaza, "Multisensor coupled spectral unmixing for time-series analysis," IEEE Trans. Geosci. Remote Sens., submitted, 2016. 